\title{
Non-rigid Atlas to Subject Registration with Pathologies for Conformal Brain Radiotherapy
}

\author{
Radu Stefanescu ${ }^{1}$, Olivier Commowick ${ }^{1,3}$, Grégoire Malandain ${ }^{1}$, \\ Pierre-Yves Bondiau ${ }^{1,2}$, Nicholas Ayache ${ }^{1}$, and Xavier Pennec ${ }^{1}$ \\ 1 INRIA Sophia - Epidaure team, BP 93, 06902 Sophia Antipolis Cedex, France \\ \{Radu.Stefanescu, Olivier.Commowick\}@sophia.inria.fr \\ 2 Centre Antoine Lacassagne, Département de Radiothérapie, 33 avenue de \\ Valombrose, 06189 NICE Cedex 2, France \\ 3 DOSISoft S.A., 45-47 Avenue Carnot, 94230 Cachan, France
}

\begin{abstract}
Warping a digital atlas toward a patient image allows the simultaneous segmentation of several structures. This may be of great interest for cerebral images, since the brain contains a large number of small but important structures (optical nerves, grey nuclei, etc.). One important application is the conformal radiotherapy of cerebral tumor, where a precise delineation of all these structures is required. However, in this case, the variability induced by the tumor or a surgical resection, that are not present in the digital atlas, prevents an accurate registration between the atlas and the patient images. Since our registration method allows to locally control the amount of regularization, we are able to explicitly introduce those areas in the warping process. For computational efficiency, we have created a parallel implementation that can be used from the clinical environment through a grid interface.
\end{abstract}

\section{Introduction}

The treatment of cerebral tumor may involve surgery, radiotherapy, or chemotherapy. Thanks to recent technological advances (on-line definition of the shape of the irradiation beam, irradiation intensity modulation during the treatment), conformal radiotherapy allows a high precision irradiation (homogeneous dose distribution within complex shapes), permitting an improvement of local control and the reduction of the complications.

This high precision radiotherapy is a powerful tool for the treatment of cerebral tumors, since the irradiation target may be close to critical structures (optical nerves, brain stem, etc.). In order to determine the best characteristics of the treatment planning, and to provide the patient follow-up, it is necessary to accurately locate all the structures of interest in the brain and the tumor. Currently, the segmentation of brain structures is manual and each structure must be delineated in each slice of a 3-D image (e.g. MRI). The treatment team spends a significant amount of time to delimit the various structures of interest with the precision requested for the conformal radiotherapy. An automatic segmentation algorithm of all the critical structures in a patient image is then an invaluable 
tool for radiotherapy, and its main requirement is a precise delineation of the structures of interest.

In order to extract all these structures in a specific patient's image, we chose to build a numerical reference atlas of all the structures we are interested in, and to use matching techniques to warp this atlas onto one patient's image. The atlas (Fig. 1b) was manually labeled from an artificial MR image (obtained from the Brainweb, see Fig. 1a). The first step is a rigid matching between atlas and the patient MRIs (usually T1, T2 and T1 injected). The recovered transformation is refined using non-rigid registration, and then applied to the atlas in order to obtain a segmentation of the patient image.

Due to its multi-subject nature, this registration problem is generally difficult. The topology of the brain, the shape of the ventricles, the number and shape of the sulci vary strongly from one individual to another. Thus, algorithms have to deal with the ambiguity of the structures to match, but they also have to take into account the large variability of the differences between the two brains.

A more important issue arises in our case with the presence of pathologies in the patient image, such as tumors or surgical resections. These structures have no equivalent in the atlas. They usually lead the non-rigid registration to important errors, especially around the pathology which is the area of interest for radiotherapy. Numerous methods and tools have been already devised to address non-rigid registration [1, but much fewer deal with pathological abnormalities.

Kyriacou et al. 2] used a biomechanical modeling of the brain and tumor based on non-linear elasticity. In the case of multi-subject patient/atlas registration, elastic models are of low relevancy, since the transformation to recover does not correspond to a physical deformation. Christensen [3] showed that in order to recover large deformations, such as the ones implied by multi-subject registration, a viscous component is needed.

Some methods 45] deal with the absence of pathology in the atlas by artificially introducing it. A first non-rigid registration between patient and atlas yields an initial deformation that is used to implant a "pathology seed" inside the atlas. This deformation is then refined by non-rigidly registering the subject image with the seeded atlas. The main problem consists in performing the first registration, which can easily fail, especially if the pathology is located closely to the brain border or the ventricles.

We have presented in [6] a non-rigid registration algorithm that uses anatomical information to locally adapt the regularization from fluid (for CSF) to viscoelastic-like (for grey/white matter). In this paper, we show how to take explicitly into account tumors and surgical resections in our framework. The first step, detailed in Section 2 consist in segmenting these areas in the patient image. Then, we recall in Section 3 the principle of the registration method, and show how the pathology is modeled as a non-informative area. Last but not least, we present in Section 4 comparative results that demonstrate the improvements brought by our method. 


\section{Segmentation of Tumors and Surgical Resections}

In order to obtain a priori information on the tumor and the surgical resection to guide the atlas registration, we have to segment these regions in the patient's brain. Thereafter, we propose two different methods for automatically delineating respectively the surgical resection and the tumor.

\subsection{Segmentation of a Surgical Resection}

A surgical resection corresponds to an absence of matter in the considered region, filled with CSF, and possibly connected with the ventricles. Its shape is more spherical than the other structures of the CSF, and is composed of only one big connected component. These are the basic properties that we exploit for delineating the resection.

First, we extract all structures behaving like CSF in the joint MR T1 and T2 histogram (low signal in T1 and high signal in T2) by fitting a 2D Gaussian on the corresponding area of the histogram. Selecting all the voxels whose joint intensity is statistically compatible gives us an oversized segmentation of CSF which still contains structures like the eyes and the ventricles. The eyes are quite easy to remove since they appear as two isolated connected components. To select them, we robustly register an atlas with an affine transformation, and remove the connected components that have an intersection with the eyes of the atlas. To separate the ventricles from the surgical resection, we use a region labeling algorithm based on a skeletonization by influence zone (SKIZ) 7]. As this labeling is sensitive to narrowings in a connected component, it easily classifies the surgical resection and the ventricle as different regions. The regions that intersect the ventricles of the atlas are removed as above.

Finally, we have to select the surgical resection region among remaining structures. The sulci are relatively small with respect to a surgical resection and thus easy to remove. The main problem comes from the possible presence of a CSF component between the brain and the skull due to brain shift during the surgical operation. The volume of this component may be quite large, but its shape is mostly flat. Thus, we compute a distance map in each remaining CSF connected component, and select the one that has the largest inscribed ball radius.

\subsection{Delineation of the Tumor}

Delineating a tumor is a hard task due to the multiple forms it may take in the image. The tumor may generate an edema at its frontiers, and contain a necrotic center. The tumor tissues and the edema usually appear like partial volume (CSF and grey matter) intensities, while the necrosis resembles the CSF.

Traditional Expectation-Maximization algorithms 8] fail to provide good results because of the presence of these tissues. An alternative is to consider tumor intensities as outliers in this mixture of Gaussians, or to add some specific classes to model the tumor and edema intensities [9]. As this was often not sufficient, some anatomical knowledge was added, either by combining geometric 
priors given by the non-rigid registration of an atlas to a tissue classification [10], or by using Markov Random Fields [11. Other methods include region growing from a region of interest delineated by one of the preceding methods using levelsets methods [12].

All these methods end up in very complex algorithm as attempt to segment all the tissues. In our case, we are only interested in the tumor segmentation, so that we could rely on a very simple mathematical morphology scheme as we developed in the previous section.

We fit this time the selected region of the joint T1 an T2 intensity histogram by a mixture of two Gaussians: one for the necrotic part of the tumor (which appear like CSF), and a second one for the tumor tissues and its edema (resembling partial volume CSF/grey matter). We obtain an oversized segmentation where we need to remove structures like the sulci or the ventricles without removing interesting parts. Indeed, we now have CSF and grey matter partial volume voxels, and the necrotic part of the tumor can be near a region containing CSF. The ventricles and the eyes are removed like before. Then the remaining part of the segmentation is labeled into SKIZ zones. Each region is then compared with an a priori statistical atlas of the CSF to compute the mean probability of belonging to the CSF. A threshold on this probability allows us to remove the CSF structures like the ventricles or the sulci. In each of these two steps we also compute a distance map to the CSF of the statistical atlas in each region to avoid removing regions containing voxels too far from the expected CSF.

\section{A Grid-Powered Registration Algorithm}

We developed in [6] an original registration method that appear particularly well fitted to our current problem. The algorithm models the transformation as a dense deformation field, which enables it to recover fine details. The degree of regularity imposed on the deformation is locally adapted in order to let the ventricles deform freely, while preserving the coherence of the brain. The user may locally tune the weight of matching versus regularization in the registration process. Finally, the resulting transformation is guaranteed to be invertible.

\subsection{General Description of the Algorithm}

Through this section, we consider $J$ to be the source image, $I$ the target image and $U=\left(U_{1}, U_{2}, U_{3}\right)$ the displacement field that transforms $J$ into $I$, so that for each point $p$, the intensity of the transformed image $(J \circ U)(p) \triangleq J(p+U(p))$ matches the one of the image $I$ at point $p$. The estimation of $U$ is twofold: first a small correction $u=\left(u_{1}, u_{2}, u_{3}\right)$ is computed by optimizing a similarity criterion, and second it is composed with $U$ before regularization.

Our registration problem is monomodal, thus the sum of squared differences is a sufficient (and adapted) measure to estimate the similarity between the images to be matched. Let the similarity criterion by $\operatorname{Sim}(I, J)=\sum_{p}(I(p)-J(p))^{2}$. A gradient descent scheme allows to optimize $\operatorname{Sim}(I, J \circ U \circ u)$, yielding a small 
correction $u$. However, this raw deformation field is usually noisy. This is particularly true in areas of the images where the intensity is uniform and the registration is mainly driven by noise. To filter out the unreliable "matches" from the raw deformation field, we use a method inspired by the image-guided anisotropic diffusion: once the gradient $u=\nabla \mathrm{Sim}$ of the similarity criterion is computed, its values are filtered using a diffusion equation: $\frac{\partial u_{i}}{\partial t}(p)=(1-k(p)) \cdot\left(\Delta u_{i}\right)(p)$, where $k(p) \in[0,1]$. The parameter $k(p)$ measures the local degree of smoothing applied to $u$, or the local confidence that we have in the similarity criterion. For $k(p)=1$, the local displacement $u_{i}(p)$ will be locally unaffected by this PDE, whereas the field is locally smoothed or even interpolated from neighboring values for $k(p)$ close to zero. For an intermediate value, this smoothing may be seen as an approximation of a viscous-elastic behavior. In practice, $k(p)$ is related to the image gradient, so that diffusion occurs in homogeneous regions.

The regularized small correction $u$ being computed, we compose it with $U$ (similarly to the regridding scheme proposed by [3]). This additionally allows for an invertible transformation $U$.

Let us now consider the regularization of $U$. Some authors used elasticity, but there are more evidences toward a visco-elastic behavior of brain material. Moreover, the resolution of these biomechanical models is quite slow. We chose a more heuristic approach that only approximates a biomechanical behavior, but which is much faster. We use the same diffusion equation than above, now with $U$, and with a stiffness field $D(p)$ (instead of $1-k(p)$ ) that now depends on the local nature of the tissues, as in [6]. Thus, combined with the above regularization, this realizes a good approximation of a visco-elastic material.

For computational efficiency, the algorithm was implemented on an inexpensive and powerful parallel machine: a cluster of workstations. However, such a cluster is more easily located in a computing center than in a clinical environment. To provide the clinical user with a user friendly interface on a visualization workstation located in its own environment, we proposed in [13] a grid service running on a parallel computer outside the clinical environment which provides on demand the computing power needed to perform the registration.

\subsection{Using A Priori Anatomical Information About the Patient}

As for every registration algorithm, we explicitly assumes that the chosen similarity metric describes a meaningful correspondence between the two images. This assumption is of course violated when the patient image contains additional structures, such as tumors or resections. Since there is no correspondent in the atlas for voxels in the "pathological" region of the patient image, we remove the influence of these voxels from the similarity metric: we assigned a null confidence to all voxels inside a dilated mask of the pathology. The dilation is necessary in order to remove the influence of the gradient caused by the pathology. As a consequence, the correspondences in this area will be determined by interpolation from non-pathological neighboring voxels, for which correspondences can be reliably estimated. We assign the pathological region the same stiffness $D$ as the surrounding tissues. 
We use the methods described in Section 2 to estimate a binary mask of the patient pathology. When performing the confidence-weighted filtering of unreliable matches, we assign a null confidence to each voxel inside the pathology. Since we specify the confidence inside the source image, we use the patient image as the source. After registration, we inverse the transformation in order to resample the atlas labeling in the subject image.

\section{Experimental Results}

Our test dataset contains 22 T1-weighted MR images of different patients. After preliminary rigid registration, the images sizes are $256 \times 256 \times 60$.

The pathology segmentation takes between 1 and 3 minute, and the non-rigid registration takes about 4 minutes on a cluster of 15 personal computers $(2 \mathrm{GHz}$ Pentium IV processors, $1 \mathrm{~GB} / \mathrm{s}$ network), which amounts to a total computation time of 5 to 10 minutes. The whole database has been processed. Results have been visually inspected by a radiotherapist, and appear satisfactory.

Figures 17 and 10 show, respectively, the atlas used for the registration, and its segmentation. The atlas has been registered with a patient image (Fig. 1:) presenting a large tumor. The pathology has been automatically segmented, and its segmentation has been introduced in the confidence field (Fig. 11). If the tumor is not taken into account in the non-rigid registration, the displacement field is biased by the tumor. This results in a false segmentation of the right lenticular nucleus and lateral ventricle (Fig. 1, g). Taking in consideration the pathology results in a interpolated displacement field in the tumor area. Therefore, the correspondences around the right lenticular nucleus and lateral ventricle are no longer biased, which leads to a better segmentation (Fig. 1f,h).

In Figure 2a, we present an example where the patient brain has a large surgical resection, that we segmented using the algorithm in Section 2 In the confidence, we assigned null values inside the resection area (see Fig. $2 \mathrm{~b}$ ). A simple non-rigid registration is not able to follow the contour of the cerebellum (see white arrow in Fig. 2r). If we use the resection segmentation in our algorithm, the segmentation is the cerebellum is largely improved (Fig. 2 $\mathrm{d}$ ).

\section{Discussion}

In this paper, we describe a non-rigid atlas to subject registration algorithm aimed at automating a brain image segmentation method for conformal radiotherapy. The main difficulty consists in the unpredictable and huge variability introduced either by the tumor or the surgical resection in the patient image, that has no correspondent in the digital atlas. These additional structures introduce false matches in the transformation, and result in a local failure of the registration around the pathology, that may also lead to errors because of the regularization. Our method is based on segmenting the pathology and reducing the weight of the voxels inside the pathology. In these regions, we locally increase the degree of regularity of the deformation field, which enables us to compute the 

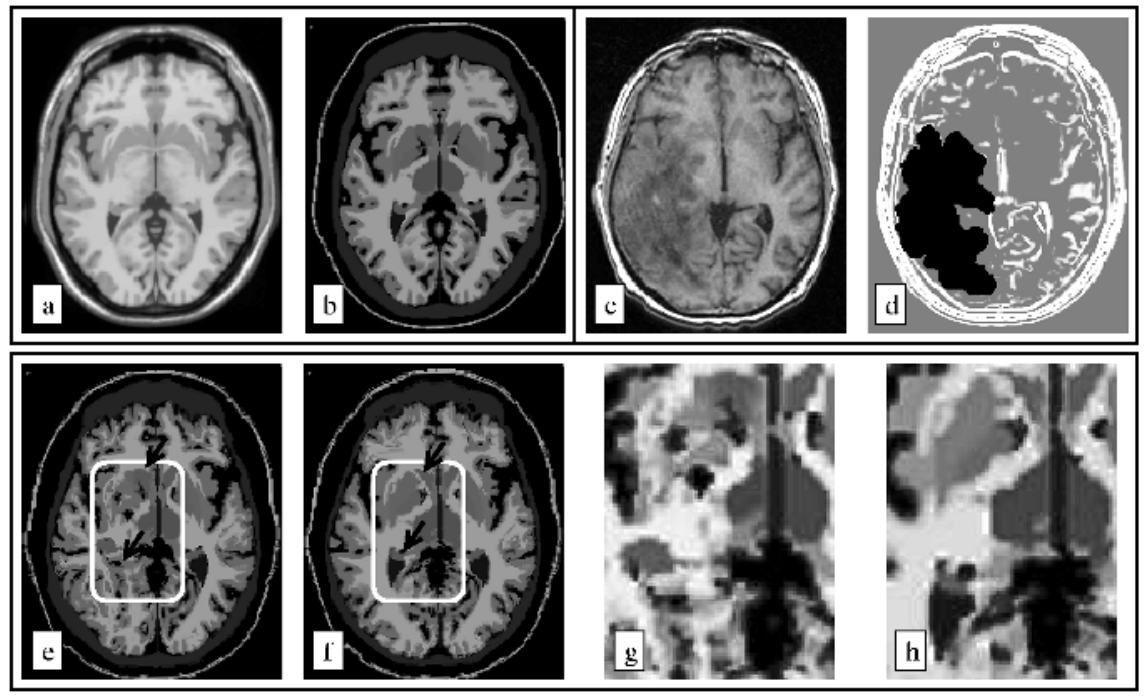

Fig. 1. Segmentation of a 3D patient image containing a large tumor. Top left: slice of atlas MRI (a) and segmentation (b). Top right: patient image (c), and confidence used for the registration (d). The confidence is 0 inside the tumor (in black on the image). Bottom line: transformation of the atlas segmentation into the patient geometry, by simple registration (e), or by taking into account the tumor (f). Fig. (g) and (h) present zooms on the same area of interest from figures (e) and (f).
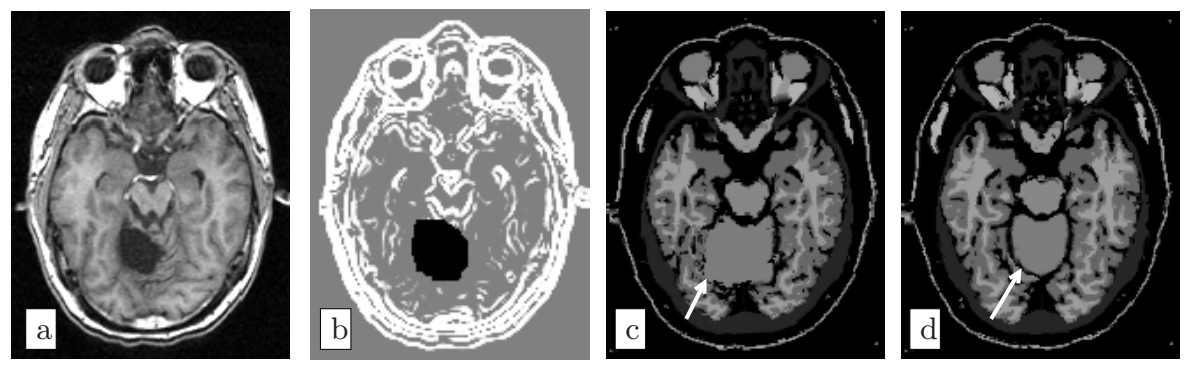

Fig. 2. Segmentation of a patient image containing a resection. (a) Patient image. (b) Confidence (resection is in black). (c) Result produced by a simple registration, un-aware of the resection. (d) Result produced by our algorithm, exhibiting a better segmentation of the cerebellum (see white arrows).

matches by interpolation. As we use a fuzzy segmentation of the pathology, the results of our algorithm gracefully degrade with the quality of the segmentation.

Results show an improvement of the segmentation in the pathology area. In the near future, we will validate this method by comparing segmentations produced by our algorithm to ones produced by clinical experts. Another future 
improvement is the use of our segmentation as an a priori for the pathology segmentation. We believe that iterating between registration and segmentation will result in future accuracy gains. In the future, we hope that automating the segmentation step will drastically reduce the time required by the conformal radiotherapy planning.

\section{References}

1. J.B.A. Maintz and M.A. Viergever. A survey of medical image registration. Medical Image Analysis, 2(1):1-36, 1998.

2. S.K. Kyriacou, C. Davatzikos, S.J. Zinreich, and R.N. Bryan. Nonlinear elastic registration of brain images with tumor pathology using a biomechanical model. IEEE Trans. Med. Imaging, 18(7):580-592, 1999.

3. G.E. Christensen, R. Rabitt, and M.I. Miller. Deformable templates using large deformation kinetics. IEEE Trans. on Image Processing, 5(10):1435-1447, 1996.

4. B.M. Dawant, S.L. Hartmann, Shiyan Pan, and S. Gadamsetty. Brain atlas deformation in the presence of small and large space-occupying tumors. Computer Aided Surgery, 7:1-10, 2002.

5. M. Bach Cuadra, J. Gomez, P. Hagmann, C. Pollo, J.-G. Villemure, B.M. Dawant, and J.-Ph. Thiran. Atlas-based segmentation of pathological brains using a model of tumor growth. In Proc. of MICCAI'02, volume 2488 of LNCS. Springer.

6. R. Stefanescu, X. Pennec, and N. Ayache. Grid enabled non-rigid registration with a dense transformation and a priori information. In Proc. of MICCAI'03, volume 2879 of LNCS, pages 804-811. Springer, 2003.

7. Pierre Soille. Morphological image analysis : principles and applications. Springer, 1999.

8. K. Van Leemput, F. Maes, D. Vandermeulen, and P. Suetens. Automated modelbased tissue classification of MR images of the brain. IEEE transactions on medical imaging, 18(10):897-908, 1999.

9. N. Moon, K. van Leemput, E. Bullitt, and G. Gerig. Automatic brain and tumor segmentation. In MICCAI, pages 372-379, 2002.

10. Michael R. Kaus, Simon K. Warfield, Arya Nabavi, Peter M. Black, Ferenc A. Jolesz, and Ron Kikinis. Automated segmentation of mr images of brain tumors. Radiology, 218(2):586-591, 2001.

11. T. Kapur. Model based three dimensional Medical Image Segmentation. Ph.d thesis, Massachusetts Institute of Technology, May 1999.

12. S. Ho, E. Bullitt, and G. Gerig. Level set evolution with region competition: Automatic 3-d segmentation of brain tumors. In Proc. 16th Int Conf on Pattern Recognition ICPR 2002, pages 532-535, 2002.

13. R. Stefanescu, X. Pennec, and N. Ayache. A grid service for the interactive use of a parallel non-rigid registration algorithm. In Proc. of HealthGrid'04. European Commission, DG Information Society, 2004. To appear in Methods of Information in Medicine. 